\section{Hämatogenes Kontaktekzem mit \\ EEM-Charakter nach beruflichem Umgang mit Pao ferro-Holz (Machaerium scleroxylon Tul.)}

\author{
C. Schulz ${ }^{1}$, B. M. Hausen ${ }^{2}$ \\ ${ }^{1}$ Universitäts-Hautklinik, Hamburg-Eppendorf \\ (Direktorin: Prof. Dr. Ingrid Moll) \\ 2 Dermatologisches Zentrum Buxtehude \\ (Chefarzt: Prof. Dr. E. W. Breitbart)
}

Zusammenfassung. Bei einem Restaurator entwickelte sich nach dem Schneiden und Bearbeiten von „Santos“-Palisander (sachgerechter Name Pao ferro), einem Ersatzholz für Rio-Palisander, ein multiformes Exanthem mit EEM-Charakter an der Brust, dem Abdomen, den Ellenbeugen, Unterarmen, im Gesicht und in den intertriginösen Bereichen. Das Holz wurde anatomisch und chemotaxonomisch als Machaerium scleroxylon Tul. identifiziert. Die Epikutantestung mit dem nur in MachaeriumArten vorkommenden Kontaktallergen (R)-3,4-Dimethoxydalbergion (Testkonzentration: $0,01 \%$ ) fiel eindeutig positiv aus. Der Restaurator reagierte darüber hinaus auch auf andere Dalbergione aus echten Palisander-Arten der verwandten Gattung Dalbergia sp., die im „Santos“-Palisander nicht vorkommen. Diese Reaktion bestätigte anamnestisch angegebene frühere Kontakte mit den genannten Nutzhölzern. Da Hautveränderungen bei der Exposition mit dem Holzstaub von Rio-Palisander (Dalbergia nigra All.) und Ostindisch-Palisander (Dalbergia latifolia Roxb.) jedoch nicht aufgetreten waren, muss eine stumme Sensibilisierung vorgelegen haben. Kreuzreaktionen auf das Chinon Primin und das mit dem (R)-4-Methoxydalbergion strukturell verwandte Obtusachinon aus Cocobolo (Dalbergia retusa Hemsl.) wurden nicht beobachtet. Allergische Kontaktekzeme mit EEM-Charakter nach Exposition gegenüber Holzstaub treten nur bei Arten mit hochgradig sensibilisierenden Inhaltsstoffen, wie z. B. dem (R)-3,4-Dimethoxydalbergion, auf.

Allergic Contact Dermatitis with Erythema MultiformeLike Lesions Due to Occupational Contact with Pao Ferro Wood (Machaerium scleroxylon Tul.). A 58-year-old restorer working with "Santos-Palisander“, a substitute for Brazilian rosewood, developed erythema multiforme-like lesions, which spread to the arms, trunk, face, neck, and intertriginous regions of the body after sawing and sanding of the timber. By wood anatomical histology and chemotaxonomical analysis the species could be identified as Machaerium scleroxylon Tul. (Pao ferro). A remarkably strong patch test reaction to diluted ( $R$ )-3,4dimethoxydalbergione (test concentration $0.01 \%$ ), the known sensitizer of Pao ferro, was obtained on day 4 . Further reactions were found after application of related dalbergiones occurring in Brazilian rosewood (Dalbergia nigra All.) and East indian rose-

Akt Dermatol 2001; 27: 198-201

(c) Georg Thieme Verlag Stuttgart · New York ISSN 0340-2541 wood (Dalbergia latifolia Roxb.). Both of them had been used by the patient previously but never caused lesions on his skin. No response occurred to obtusaquinone from Cocobolo (Dalbergia retusa Hemsl.) and primin from Primula obconica L. Simultaneous exanthematous, haemorrhagic and EEM-like eruptions do only occur after contact with wood dust of species containing very strong sensitizers such as (R)-3,4-dimethoxydalbergione.

\section{Einleitung}

Hautveränderungen mit EEM-ähnlichem, hämorrhagischem und hämatogenem Charakter sieht man gelegentlich bei Kontakt mit Allergenen, die ein sehr hohes Sensibilisierungsvermögen besitzen. Neben Allergenen aus industriellen Produkten des täglichen Umgangs kommen auch Inhaltsstoffe von Pflanzen und Nutzhölzern als Ursache in Frage. Das als Ersatz für echtes Rio-Palisander (Dalbergia nigra All.) früher häufiger, heute noch gelegentlich eingesetzte oder als Irrläufer auf den Markt gelangende Holz des Pao ferro-Baumes Machaerium scleroxylon Tul. (Familie: Leguminosae-Papilionaceae) zeichnet sich durch seine unrühmliche Eigenschaft aus, ein solches, stark sensibilisierendes Kontaktallergen zu enthalten. Beschreibungen von stark ausgeprägten Dermatitiden liegen nicht nur aus Europa [1-6] und den USA [7], sondern auch aus dem Ursprungsland Brasilien vor [8]. Um seine Ähnlichkeit mit dem Rio-Palisander hervorzuheben und seinen Absatz zu erhöhen, wird das in seinen physikalischen Eigenschaften aber auch in seiner Maserung sehr ähnliche Holz häufig unter der Bezeichnung „Santos“- und „Peru“-Palisander oder „Palisander Rosé“ im Handel angeboten. Eine Verwechslung mit dem echten Palisander ist nicht nur möglich, sondern wahrscheinlich auch beabsichtigt, um von der seit langem bekannten gesundheitsschädigenden Wirkung $[9,10]$ dieser zwar botanisch verwandten, aber chemotaxonomisch deutlich unterscheidbaren Holzart abzulenken. Dass sie billiger und leichter zu beschaffen ist (z.B. aus Bolivien), trägt sicher ebenfalls zu ihrem mengenmäßig geringeren, dafür aber stetigen Auftauchen auf dem Markt bei. Die brasilianischen Eigennamen, z. B. Caviuna vermelha, Morado, Moradillo und Pao ferro finden daher bei Importware so gut wie keine Verwendung. Seine Verwendung erstreckt sich auf alle auch für die echten Palisanderarten geltenden Bereiche: z. B. Möbel, Musikinstrumente, Furniere, Drechselarbeiten, Griffe, Gehäuse von Fernsehgeräten, Intarsien, Rahmen, Regale, Schränke, Vitrinen, Präzisionsinstrumente. 
Über einen neuen, kürzlich beobachteten Fall möchten wir im Folgenden berichten.

\section{Kasuistik}

Ein 58-jähriger Restaurator schneidet und bearbeitet seit einiger Zeit eine als „Santos“-Palisander bezeichnete Holzart, die er in Form von Bohlen für die Restauration einer großen Bücherwand auf Wunsch eines Kunden kaufte. Eine Absauganlage fehlt, so dass die Späne und der feinere Holzstaub an den Unterarmen, im Hemdausschnitt und im Gesicht auf die Haut gelangen. Zur Belüftung öffnet er die Fenster, gelegentlich verwendet er eine Maske. Drei Tage nach Beginn der Arbeit entwickeln sich bis handtellergroße, teils konfluierende, symmetrisch angeordnete, lividrötliche, scharf begrenzte Maculae, die zum Teil auch kokardenartig imponierend auf der Brust, im Gesicht, am Abdomen sowie an den Unterarmen und in den Ellenbeugen auftreten. Die Hände sind frei (Abb.1). Es besteht ein starker Juckreiz. Nach der Vorstellung in der Klinik im Mai 2000 und einer ersten Verdachtsdiagnose über die wahrscheinliche Ursache, arbeitet der Patient an einem Sonntag jedoch weiter und stellt sich am Montag wieder vor, da sich die Hautveränderungen nun auf das übrige Integument (Hals, Nacken, Axillen, Thorax, Bauch, Rücken, Stirn und auch nasolabial) ausgebreitet haben. Neben dem Juckreiz stellt sich nun auch ein Brennen ein. Die Therapie erfolgt systemisch mit $80 \mathrm{mg}$ Methylprednisolon, langsamer Reduktion auf $40 \mathrm{mg}$ und anschließend lokal mit Methylprednisolonaceponat in einer Creme. Nach Ausklingen der Behandlung und Meiden des Holzes Abheilung. Der Patient gibt an, bereits früher mit Palisander und anderen Holzarten gearbeitet, aber nie derartige Hautveränderungen beobachtet zu haben.

\section{Allergologische, histologische und chemische Untersuchungen}

Der mikroskopische Befund einer Gewebeprobe aus dem Abdomenbereich zeigte eine Spongiose mit Einwanderung von einzelnen Lymphozyten, subepidermal ein leichtes Ödem der papillären Dermis sowie eine lymphohistiozytäre Infiltration mit einzelnen neutrophilen und eosinophilen Granulozyten.

Die vom Patienten mitgebrachte Holzprobe war sowohl holzanatomisch (Stirnholzstruktur im Anschnitt unter dem Mikroskop) als auch durch eine chemische Analyse der Inhaltsstoffe leicht als Machaerium scleroxylon Tul. zu identifizieren. Der über Nacht bei Zimmertemperatur aus handgefertigten Spänen (um eine Zerstörung des Allergens durch Überhitzung zu vermeiden) hergestellte Ethanolextrakt wurde im Laufmittelsystem Chloroform-Methanol $(100+1)$ auf einer Dünnschichtplatte (Kieselgel 60, 0,25 mm dick, mit Fluoreszenzindikator MERCK) chromatographisch aufgetrennt (Dünnschichtchromatographie = DC). Zum Vergleich diente authentisches $(\mathrm{R})$ 3,4-Dimethoxydalbergion [= (R)-3,4-DMD] (Abb. 2) aus einer früheren Untersuchung sowie ein wie oben hergestellter Ethanolextrakt aus einem einwandfrei als $M$. scleroxylon identifizierten, bereits vorliegenden Holzmuster. Der auf gleicher Laufhöhe mit dem (R)-3,4-DMD unter dem UV-Licht bei $254 \mathrm{~nm}$ erscheinende Fleck ließ sich mit dem Craven-Test [11] blau anfärben (Nachweis für ein Chinon mit mindestens einem freien C-Atom am chinoiden Ring). Auch die als Hydrochinon vorliegende Vorstufe des (R)-3,4-DMDs war auf der DCPlatte bei $366 \mathrm{~nm}$ als großer Fluoreszenzfleck deutlich zu erkennen.

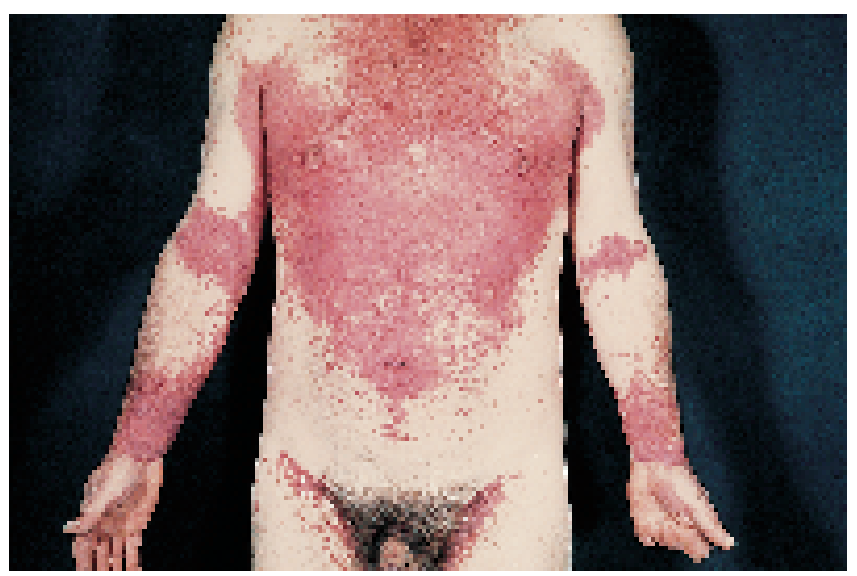

Abb. 1 Hämorrhagische und EEM-ähnliche Veränderungen nach Einwirkung von Pao ferro-Holzstaub.
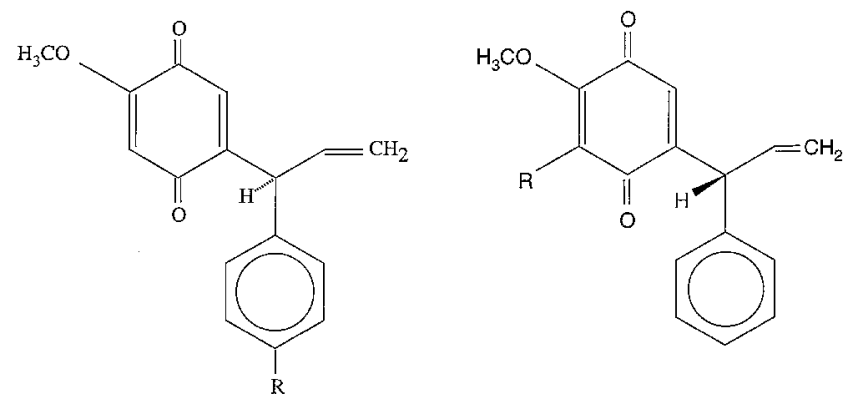

Abb. 2 Strukturen der Dalbergione. (S)-4-Methoxydalbergion $(\mathrm{R}=\mathrm{H})$; (S)-4,4'-Dimethoxydalbergion $\left(\mathrm{R}=\mathrm{OCH}_{3}\right)$; $(\mathrm{S})$-4'-Hydroxy-4methoxydalbergion $(\mathrm{R}=\mathrm{OH})$; $(\mathrm{R})$-4-Methoxydalbergion $(\mathrm{R}=\mathrm{H})$; $(\mathrm{R})$ 3,4-Dimethoxydalbergion ( $\left.\mathrm{R}=\mathrm{OCH}_{3}\right)$. Links: (S)-Form, rechts: (R)Form.

Tab. 1 Ergebnisse des Epikutantests

\begin{tabular}{llll}
\hline Allergen & Konzentration & $24 \mathrm{~h}$ & $72 \mathrm{~h}$ \\
\hline (R)-3,4-Dimethoxydalbergion & $0,01 \%$ & + & ++ \\
(R)-4-Methoxydalbergion & $1 \%$ & + & ++ \\
(S)-4-Methoxydalbergion & $1 \%$ & + & ++ \\
(S)-4'-Hydroxy-4- & $1 \%$ & $\varnothing$ & $\varnothing$ \\
methoxydalbergion & $1 \%$ & & \\
(S)-4,4'-Dimethoxydalbergion & $1 \%$ & + & + \\
Obtusachinon & $0,1 \%$ & $\varnothing$ & $\varnothing$ \\
Primin & $0,01 \%$ & $\varnothing$ & $\varnothing$ \\
\hline
\end{tabular}

Für die Epikutantestung (August 2000) zogen wir das (R)-3,4$\operatorname{DMD}(0,01 \%)$ sowie verwandte Dalbergione (alle in $1 \%$ iger Konzentration) aus den verschiedenen Palisanderarten, mit denen der Patient früher Kontakt hatte, heran (siehe Tab.1). Um Kreuzreaktionen auszuschließen, prüften wir auch das Benzochinon Primin aus der Becherprimel. Die Testergebnisse der Tab. 1 zeigen eindeutig eine ++-plus-Reaktion auf das stark sensibilisierende (R)-3,4-Dimethoxydalbergion (Testkonzentration $0,01 \%$; !) sowie gleichstarke Testantworten auf die Hauptallergene des Rio-Palisanders (Dalbergia nigra All.) und 
des Ostindischen Palisanders (Dalbergia latifolia Roxb.) - (R)4-Methoxydalbergion und (S)-4-Methoxydalbergion (alle 1\%). Das in beiden Arten vorkommende Nebenallergen (S)-4,4'-Dimethoxydalbergion erreichte nur ein +-plus (Abb. 2). Positive Reaktionen auf das Obtusachinon aus dem Cocobolo-Holz (Dalbergia retusa Hemsley) und das Primin aus der Primula obconica L. blieben aus.

\section{Diskussion}

Die Ergebnisse der Epikutantestung passen sehr gut zu der stark ausgeprägten Sensibilisierung gegenüber dem (R)-3,4Dimethoxydalbergion aus dem Pao ferro-Holz. Reaktionen mit EEM-Charakter, lividroter Färbung und Befall jener Körperstellen, die primär mit dem feinen Holzstaub in Berührung kommen, sind typisch für diese Holzart $[1,13,14]$. Dass es bei weiterer Exposition zu Streuungen auf andere Körperstellen, z.B. die Leisten, den Genitalbereich (Abb.3) und schließlich auf das gesamte Integument kommt, ist mehrfach beobachtet worden $[2,3,12]$. In intertriginösen Körperregionen wird der feine Staub durch den Schweiß besonders leicht aufgeweicht und gibt dem Allergen den Weg in die Haut frei. Gleichzeitig wird dabei die Vorstufe (das Hydrochinon des [R]-3,4-DMDs) oxidativ in das wirksame Chinon umgewandelt [1]. Direkter Kontakt mit Fertigprodukten benötigt etwas längere Latenzzeiten bis zum Eintritt einer Sensibilisierung (Abb.4). Doch kann bei empfindlicher Haut, wie sie in den Interdigitalräumen der Finger vorliegt, bereits nach wenigen Wochen eine lividrote Färbung als Zeichen der erworbenen Kontaktallergie beobachtet werden (Abb. 5).

Kreuzreaktionen auf die anderen Dalbergione aus den echten Palisanderarten sind trotz der strukturchemischen Verwandtschaft (Abb. 2) bisher nicht schlüssig zu beweisen. Der Restaurator könnte sich also bei seinen früheren Arbeiten mit dem echten Palisander stumm sensibilisiert haben, da er beim Arbeiten mit diesem keine Hautveränderungen entwickelte. Den Beweis hierfür liefern die deutlich positiven Testreaktionen auf das (R)-Methoxydalbergion und (S)-4-Methoxydalbergion sowie die schwächere Antwort auf das (S)-4,4'-Dimethoxy-dalbergion. Keines dieser Dalbergione kommt im Pao ferroHolz vor. Diese Allergene haben jedoch der Sensibilisierung gegenüber dem (R)-3,4-DMD mit hoher Wahrscheinlichkeit den Weg geebnet, so dass der Patient bereits nach dem ersten Kontakt die geschilderten Hautveränderungen entwickelte. Andererseits ist nicht auszuschließen, dass er bereits vorher schon unwissentlich mit Pao ferro gearbeitet, aber noch nicht darauf reagiert hat. Irrläufer sind nicht selten, eine Verwechslung mit echtem Palisanderholz leicht möglich und ein Ersatz von Rio-Palisander durch Lieferung von Pao ferro mit der Handelsbezeichnung „Santos“-Palisander geschieht häufig [2,3, $5,6,13-15]$.

Allergische Kontaktekzeme mit multiformem Erscheinungsbild, d.h. hämorrhagischer, hämatogener und EEM-ähnlicher Ausprägung, kommen aufgrund der meist sehr hohen Konzentration des starken Sensibilisators (R)-3,4-DMD zustande. Diese liegt im Durchschnitt bei 1-2\%, kann aber in einigen Fällen bis zu > 6\% ausmachen. Quantitative Analysen der Holzproben aus sieben verschiedenen Ländern im Jahre 1983 und 1984 [16,17] ergaben Konzentrationen zwischen $1 \%$ und 4,9\%, aus Dänischen Furnier- und Fernsehgerätewerken des Jahres 1987 sogar bis zu 6,12\% [18]. Da die subirritative Testkonzen-

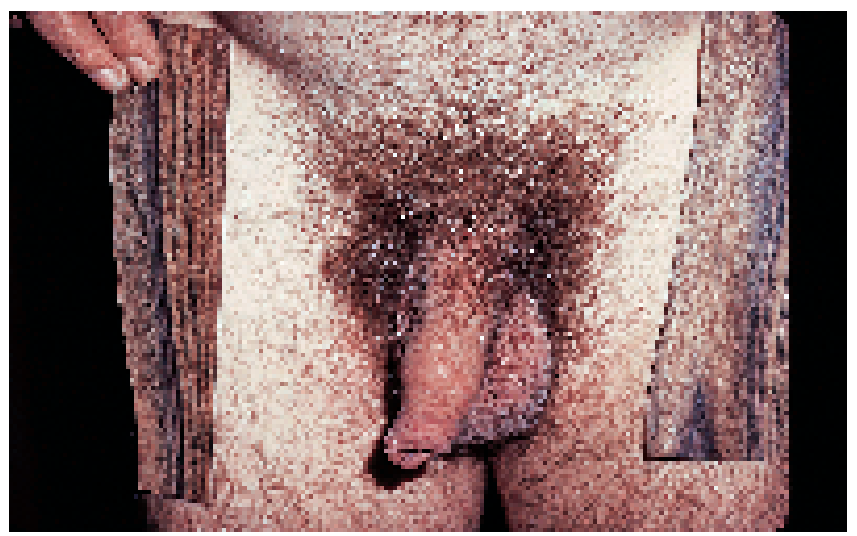

Abb. 3 Hautveränderungen durch Pao ferro-Holz im Genitalbereich (aus einem unveröffentlichten Fall).

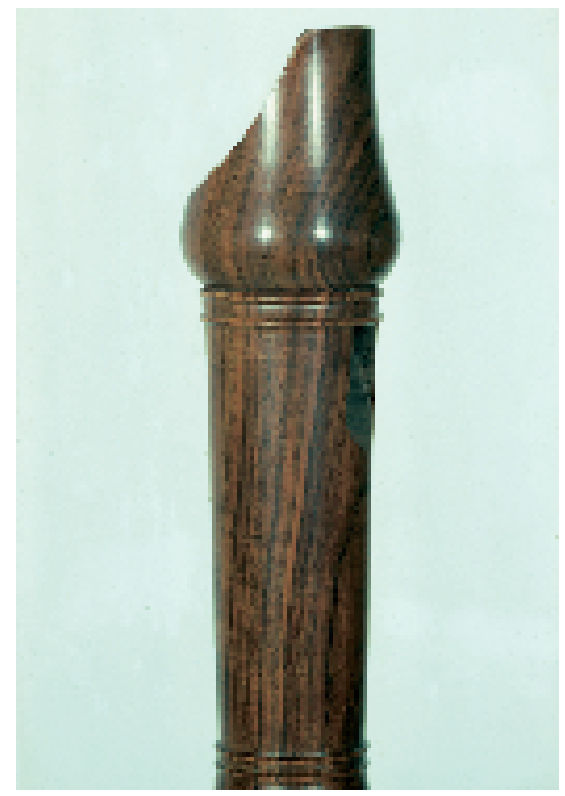

Abb. 4 Blockflöte aus Machaerium scleroxylon-Holz.

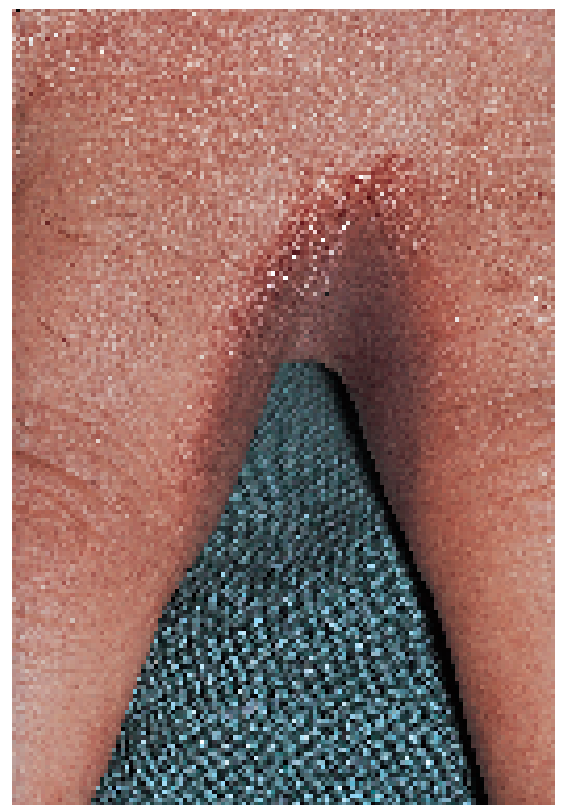

Abb. 5 Lividroter Fingerzwischenraum bei einem Furnierprüfer (aus [3]). 
tration bei 0,01\% liegt, haben wir es hier in der Regel mit der Überlagerung einer irritativen und allergischen Reaktion zu tun. Im Holzstaub liegt jedoch nur ein Teil des Dalbergions in chinoider Form vor. Es entsteht bei der Bearbeitung des Holzes aus der Vorstufe durch Wärmeeinwirkung und durch Oxidation an der Luft. Gelangt die Vorstufe mit dem feinen Holzstaub auf die Haut, setzt sich die Oxidation dieser Vorstufe über die Enzyme der Haut weiter fort. Der Anteil des Hydrochinons im Holz beträgt das 3,5fache des Chinons [1]. Würde man die gesamte Menge des als Vorstufe vorliegenden (R)-3,4-DMDs oxidativ umwandeln und prozentual berechnen, käme man leicht über die $10 \%$-Grenze hinaus.

(R)-3,4-DMD ist ein hochgradiges Irritans und ein sehr starker Sensibilisator. Seine experimentell bestimmte Sensibilisierungspotenz kommt der des Primins sehr nahe [19]. Sie beträgt das 13fache der Sensibilisierungskapazität der anderen Dalbergione [19]. Eine direkte Testung mit dem Holzstaub sollte daher unbedingt unterbleiben. Es kann leicht zu einer aktiven Sensibilisierung kommen [7], auch bei Kontrollpersonen [20].

Liegen große Mengen des Allergens im verwendeten Holz vor, ist bei der bekannten hohen Sensibilisierungspotenz mit einer kurzen Latenzzeit und ausgesprochen heftigen Reaktionen zu rechnen. Wie in diesem und in früheren Fällen beobachtet, treten Streuphänomene mit EEM-ähnlichem Charakter auf $[2,3,14,21,22]$. Unter allen Nutzhölzern aus tropischen Gebieten kommt dem Pao ferro-Holz, trotz der geringen Importmengen, auch heute noch ein nicht zu unterschätzendes allergologisches Risiko zu.

Machaerium scleroxylon wird von der „Senatskommission zur Prüfung gesundheitsschädlicher Arbeitsstoffe der Deutschen Forschungsgemeinschaft“ seit 1995 mit der Bezeichnung „S“ (für sensibilisierend) gekennzeichnet [23].

\section{Danksagung}

Frau U. Stoczek, Markgröningen, gebührt Dank für die Übersetzung der slowakischen Arbeit.

\section{Literatur}

${ }^{1}$ Morgan JWW, Orsler RJ, Wilkinson S. Dermatitis due to the wood dust of Khaya anthotheca and Machaerium scleroxylon. Br J Indust Med 1968; 25: 119-125

${ }^{2}$ Conde-Salazar L, Garcia Diez A, Rafeensperger F, Hausen BM. Contact allergy to the Brazilian rosewood substitute Machaerium scleroxylon Tul. (Pao ferro). Contact Dermatitis 1980; 6: 246-250

${ }^{3}$ Hausen BM. Häufigkeit und Bedeutung toxischer und allergischer Kontaktdermatitiden durch Machaerium slceroxylum Tul. (Pao ferro), einem Ersatzholz für Palisander (Dalbergia nigra All.). Hautarzt 1982; 33: $321-328$

${ }^{4}$ Sâk M, Uhrik J, Fabian J, Strawenska H, Hermanova E . Dermatózy vyvolané drevinom Machaerium scleroxylon. Cšl Dermatol 1983; 58: 89-91

${ }^{5}$ Jacobsen FK, Schmidt H, Brandrup F. Toksiske og allergiske reaktioner forårsaget of Machaerium scleroxylum Tul. på en møbelfabrik. Ugeskr Læger 1987; 149: 219-220

${ }^{6}$ Ibsen HHW, Larsen A, Jespen JR, Brandrup F, Edvardsen KM. Arbeijdsbetinget kontaktdermatitis fremkaldt of træarten Machaerium scleroxylum. Ugeskr Læger 1987; 149: 244-245
${ }^{7}$ Maibach HI. Active sensitization to wood extracts. Contact Dermatitis Newsletter 1970; 7: 149

${ }^{8}$ Miranda Bastos AD, Matos Filho AD. A ,Jacaranda“ timber causing dermatitis. Proc Fifth Forest Congr (Univ of Seattle, Washington) 1962; 3: $1414-1416$

${ }^{9}$ Großmann J. Gesundheitsschädigende Hölzer. Bayer Industr Gewerbeblatt 1910; 96: $51-56$

${ }^{10}$ Freise FW. Gesundheitsschädigungen durch Arbeiten mit giftigen Hölzern. Beobachtungen aus brasilianischen Gewerbebetrieben. Arch Gewerbepath u -hygiene 1932; 3: 1-14

${ }^{11}$ Craven R. A sensitive colour reaction for certain quinones. J Chem Soc 1931: 1605 - 1606

12 Beck MH, Hausen BM, Dave VK. Allergic contact dermatitis from Machaerium scleroxylum Tul. (Pao ferro) in a joinery shop. Clin Exper Dermatol 1984; 9: 159-166

${ }^{13}$ Woods B. Contact dermatitis from „Santos“ rosewood. Contact Dermatitis 1987; 17: 249-250

${ }^{14}$ Irvine C, Reynolds A, Finlay AY. Erythema multiforme-like reaction to „rosewood“. Contact Dermatitis 1988; 19: 224-225

${ }^{15}$ Chieregato C, Vincenzi C, Guerra L, Rapacchiale S. Occupational airborne contact dermatitis from Machaerium scleroxylon. Contact Dermatitis 1993; 29: 164-165

${ }^{16}$ Hausen BM. Ungewöhnliche Kontaktsensibilisierung im Konditorgewebe. Akt Dermatol 1984; 10: 13-16

${ }^{17}$ Hausen BM. Aktuelle Kontaktallergene. Allergologie 1983; 6: $194-197$

18 Roed-Petersen J, Menné T, Nielsen KM, Hjorth N. Is it possible to work with Pao ferro (Machaerium scleroxylum Tul)? Arch Dermatol Res 1987; 279: 108 - 110

${ }^{19}$ Schulz KH, Garbe I, Hausen BM, Simatupang MH. The sensitizing capacity of naturally occurring quinones. Part II. Benzoquinones. Arch Dermatol Res 1979 ; 264: 275-286

${ }^{20}$ Hausen BM. Contact allergy to woods. In: Guin JD, Beaman JH (Eds). Clinics in Dermatology. Vol 4. Philadelphia: Lippincott, 1986

${ }^{21}$ Holst R, Kirby J, Magnusson B. Sensitization to tropical woods giving erythema multiforme-like eruptions. Contact Dermatitis 1976; 2: $295-296$

${ }^{22}$ Shimizu S, Chen KR, Pratchyapruit WO, Shimizu H. Tropical wood-induced bullous erythema multiforme. Dermatology 2000; 200: 59-62

${ }^{23}$ Gesundheitsschädliche Arbeitsstoffe. Toxikologisch-arbeitsmedizinische Begründungen von MAK-Werten. 21. Lieferung: Machaerium scleroxylon. Weinheim, Bergstraße: Verlag Chemie, 1995: $1-3$

\section{Dr. C. Schulz}

Artemed Fachklinik

Mozartstraße 14a-16

80336 München

\section{Prof. Dr. B. M. Hausen}

Dermatologisches Zentrum

Elbe Klinikum Stade Buxtehude

Am Krankenhaus 1

21614 Buxtehude 